\title{
Treatment Targets in Intracerebral Hemorrhage
}

\author{
Navdeep Sangha $\cdot$ Nicole R. Gonzales
}

Published online: 6 July 2011

(C) The American Society for Experimental NeuroTherapeutics, Inc. 2011

\begin{abstract}
Intracerebral hemorrhage (ICH) imparts a higher mortality and morbidity than ischemic stroke. The therapeutic interventions that are currently available focus mainly on supportive care and secondary prevention. There is a paucity of evidence to support any one acute intervention that improves functional outcome. This chapter highlights current treatment targets for ICH based on the pathophysiology of the disease.
\end{abstract}

Keywords Intracerebral hemorrhage · Pathophysiology Treatment $\cdot$ Clinical trials $\cdot$ Stroke

\section{Introduction}

Intracerebral hemorrhage (ICH) accounts for approximately 10 to $15 \%$ of all strokes that occur in the United States, Europe, and Australia, as well as 20 to $30 \%$ of all strokes that occur in Asian countries [1, 2]. ICH is more common in men than in women [3] and varies according to ethnic background. Individuals of African, Japanese, and Hispanic descent have a higher risk of ICH [4-6]. Blacks and Hispanics have been found to have double the risk of ICH compared to Caucasians $[4,6]$. ICH continues to impart a considerable degree of morbidity and mortality, with 30-day mortality ranging from 44 to $50 \%[1,7,8]$. ICH remains a devastating disease and current treatment options lag far behind those for ischemic stroke.

Electronic supplementary material The online version of this article (doi:10.1007/s13311-011-0055-z) contains supplementary material, which is available to authorized users.

\footnotetext{
N. Sangha $\cdot$ N. R. Gonzales $(\triangle)$

Department of Neurology,

University of Texas Medical School-UT Health,

6431 Fannin, MSB 7.118,

Houston, TX 77030, USA

e-mail: nicole.r.gonzales@uth.tmc.edu
}

There is evidence that advancements in specialized care, such as neurological intensive care units [9], and neurointensivists [10] impact outcomes in patients with $\mathrm{ICH}$; however, there are no approved therapies that improve outcome, and treatment remains mainly supportive. Despite the lack of available acute treatment options for ICH, the last decade of clinical and preclinical research has identified important concepts in its pathophysiology, and how this information might be used in developing treatment. Recent clinical trials in ICH have identified important considerations in patient selection, which have provided information for current and future trials in evaluating treatment for $\mathrm{ICH}$, and preclinical work in $\mathrm{ICH}$ has identified new treatment targets.

It is intuitive that an understanding of the pathophysiology of the disease provides a starting point for identification of treatment targets. The pathophysiology of $\mathrm{ICH}$ begins with the predisposition of developing the disease. This includes genetics and risk factors that conspire to generate the ictus. Once present, ICH causes both primary and secondary injury. The primary insult is due to disruption of adjacent tissue and mass effect [11]. Secondary injury occurs with the development of edema, free radical formation, inflammation, and direct cellular toxicity due to the deposited hematoma and subsequent degradation byproducts. Each of these phases of disease provides a potential treatment target. The multiple steps along the path of disease also highlight the fact that successful treatment for ICH will most likely be multifaceted with different treatments at different time points.

\section{ICH Genetics}

The most common causes of spontaneous ICH include hypertension and cerebral amyloid angiopathy. However, anticoagulation associated $\mathrm{ICH}$ is increasing in incidence [12]. Warfarin use increases the risk of ICH 2 to 5 times, 
depending on the intensity of anticoagulation $[13,14]$. The risk of ICH with anticoagulation may also depend on an individual's race. Asians have a 6 times higher risk on warfarin compared to whites and are twice as likely to have an ICH when compared to blacks and Hispanics [15].

The genetics of ICH is complicated as there are multiple environmental risk factors that interact with complex traits, such as hypertension, to increase the risk of ICH; however, there are also other well-defined genetic associations, which are risk factors for ICH with characteristic patterns. Although a complete discussion regarding the current status of ICH genetics is beyond the scope of this chapter, in this section we will discuss areas in which genetic data has or will provide information for future treatment efforts.

\section{Cerebral Amyloid Angiopathy}

Cerebral amyloid angiopathy (CAA) is a major risk factor for ICH with a recurrence rate of $10.5 \%$ per year [16]. CAA is age dependent, uncommonly occurring in individuals $<60$ years of age [17]. The most common locations of hemorrhage due to CAA are the cerebral and cerebellar cortices, in contrast to the deeper locations associated with hypertension. The deposition of protein consisting of amyloid in the tunica media and adventitia of the capillaries and arteries of the brain is the primary pathological feature of CAA. These amyloid deposits are similar to those seen in Alzheimer's disease. The amyloid-beta fragment is the primary component of the plaques $[18,19]$.

The pathogenesis behind the deposition of the beta amyloid peptide into cerebral blood vessels and its increasing incidence with age has not been clearly defined. There is, however, a relationship between apolipoprotein E (APOE) and CAA. APOE is essential for the normal catabolism of triglyceride-rich lipoprotein constituents. Individuals carrying the APOE epsilon 2 $(\varepsilon 2)$ or epsilon $4(\varepsilon 4)$ alleles seem to be at greater risk for CAA-related hemorrhage than those with only the APOE epsilon $3(\varepsilon 3)$ allele, which is more common in the population $[20,21]$. This connection has been clearly defined in Caucasian populations, but is not necessarily as clear in other ethnicities due to lack of evidence or a poor association as seen in the Japanese population [22]. Studies comparing normal controls against those with pathology positive CAA reveal that both APOE $\varepsilon 2$ and $\varepsilon 4$ are present in two-thirds of patients with CAA compared to one-fourth of those without. Individuals who have both alleles have an earlier onset of disease and an increased risk of recurrence $[16,21,23]$. Both APOE $\varepsilon 2$ and $\varepsilon 4$ are independent risk factors for lobar ICH, however, in the same large-scale genetic association study, APOE $\varepsilon 4$ was also found to be associated with deep ICH [24]. Finally, there is now evidence that APOE $\varepsilon 2$ may be associated with increased hematoma volumes at presentation, as well as subsequent hematoma expansion, which has important prognostic implications [25].

The pathogenesis of the hemorrhage associated with CAA begins with the deposition of amyloid beta-peptide into the wall of capillaries and arterioles. This deposition initiates changes of the blood vessel resulting in decreased integrity of the vasculature. There may be a synergistic effect of APOE in carriers of both the $\varepsilon 2$ and $\varepsilon 4$ alleles. The presence of the $\varepsilon 4$ and $\varepsilon 2$ genes may result in increased vulnerability of amyloid laden blood vessels. The $\varepsilon 4$ allele has been demonstrated to increase the amyloid deposition [26], whereas the $\varepsilon 2$ allele can induce necrosis of blood vessels with amyloid deposits [23].

The management of CAA lies mainly in supportive measures, and there is no evidence to support one specific therapy to help reduce the risk of recurrent hemorrhage. However, the use of proteoglycans and compounds that mimic the glycosaminoglycan moieties of proteoglycans have been observed to associate with amyloid fibril deposits in vitro [27]. These deposits have been implicated in the polymerization of amyloid proteins and the propagation of the deposition process. Recently a phase II study, Cerebril $^{\mathrm{TM}}$ in Patients With Lobar Hemorrhage Related to Cerebral Amyloid Angiopathy, evaluating the safety of a glycosaminoglycan mimic moiety was completed [28]. The results of this study are pending, and if they are positive, they may lead to a larger phase III efficacy study in patients with CAA.

\section{COL4A1}

COL4A1 is located on chromosome 13q34 and encodes the $\alpha 1$ chain of type IV collagen, which is a basement membrane protein. Mutations in COL4A1 disrupt the basement membrane and weaken the blood vessel [29]. This mutation has been linked to a spectrum of cerebral small-vessel disease in humans, including perinatal ICH with consequent porencephaly [30], adult-onset $\mathrm{ICH}$, microbleeds, lacunar strokes, and leukoaraiosis, and follows an autosomal dominant pattern of inheritance [31]. Mice with the COL4A1 mutation have hemorrhage associated with birth trauma, as well as clinically asymptomatic cerebral hemorrhage. The clinical phenotype in a family with this mutation was variable, demonstrating retinal arterial tortuosity, migraine with aura, infantile hemiparesis, leukoencephalopathy, and microbleeds $[29,32]$. These findings suggest that a genetic predisposition combined with environmental stress (i.e., trauma, oral anticoagulant use, hypertension) may increase the risk of ICH [29]. This information may be important in determining ICH etiology in those patients without traditional ICH risk factors and it could have important preventive implications [33]. 
Technological advancements in the genetic evaluation of disease have transformed the scope and nature of genetic data that can now be obtained to understand a complex disease such as ICH. Researchers are now able to conduct accurate and efficient analyses across the entire genome of a study subject. Specifically, the genome-wide association study (GWAS) represents an experimental framework that can rapidly and efficiently identify novel pathogenic mechanisms, and can confirm a role for previously known mechanisms [34].

At present, GWAS studies of ICH among whites are ongoing. In the largest GWAS of ICH reported to date, no association with ICH was identified after multiple comparisons. This is consistent with ICH having a complex etiology with multiple risk factors [35]. Despite these advances, there is a critical lack of ICH cases among minorities with DNA available for genotyping, which limits our understanding of the epidemiology of $\mathrm{ICH}$ among minorities. Given the numerous risk factors to be examined and the potential for interactions, a large sample size is required, and no single center would be able to efficiently recruit sufficient numbers of cases and controls. The Ethnic/Racial Variations of Intracerebral Hemorrhage (ERICH) study aims to address this gap in the literature. This multicenter study will enroll 1000 cases of ICH among whites, 1000 cases of ICH among blacks, and 1000 cases of ICH among Hispanics. In addition, 3000 controls will be matched to cases by race/ethnicity, age, gender, and geographic location. The study began in 2010 and is expected to be complete in 2015 [36].

\section{Primary Injury}

Primary ICH or spontaneous ICH is often believed to either be due to hypertension or CAA. Other risk factors for primary ICH include older age, alcohol consumption, and smoking [37]. The subsequent section will focus on hypertensive ICH because CAA was previously discussed.

Chronic hypertension is the most common modifiable risk factor for ICH. Hypertension pre-dating the hemorrhage has been found to be present in 45 to $56 \%$ of individuals, depending on the definition applied in the study. As one ages, the risk for ICH increases as an independent factor [38]. However, older age in conjunction with hypertension further increases an individual's risk [39]. Additional evidence supporting hypertension as an etiological factor is seen with improved control of hypertension leading to a decreased incidence of ICH [37].

Hypertensive hemorrhages are most commonly located in the putamen, thalamus, pons, and cerebellum; however, lobar hemorrhages are also frequently caused by hypertension $[40$, 41]. The most common belief regarding the etiopathogenesis of hypertensive-associated hemorrhages is due to the rupture of microaneurysms formed by chronic hypertension, which was supported by postmortem studies [42, 43]. The alternative explanation suggested that the pathology of the vascular change was due to true dilatations of the arterial wall; however, evidence emerged in the early 20th century that the pathological changes may actually be due to a dissection in the arterial wall leading to a pseudoaneurysmal dilatation [44]. This dissection may be due to chronic hypertensive induced intimal hyperplasia with vessel-wall hyalinosis leading to focal fibrinoid necrosis. The subsequent endothelial damage is also associated with subadventitial hemorrhages and extravascular clots. Continuous leaking of small amounts of blood from the pseudoaneurysm has been documented. The final step in the cascade leading to the actual ICH may be due to the breakdown in the balance between the clotting system and the rate of bleeding from the pseudoaneurysm $[45,46]$.

Ultimately, the intervention most likely to impact disease is the prevention of complications of hypertension; however, an understanding of the pathology related to hypertensive $\mathrm{ICH}$ may play a role in the downstream events that will influence hematoma size, expansion, and resolution.

\section{Clinical Studies Targeting Hematoma Expansion}

The evolution of the hematoma after initial hemorrhage has been well-described. The majority of the initial neurological deficit is due to the hematoma. Previously, early neurological decline was attributed to the development of edema; however, several authors have shown that early decline in neurological status can also be attributed to hematoma expansion (HE) [47-51]. One study enrolled 103 patients with ICH and performed computed tomographic (CT) scans 3 hours after symptom onset and follow-up computed tomographic scans after $1 \mathrm{~h}$ and $20 \mathrm{~h}$. There was a $33 \%$ enlargement in $26 \%$ of patients at the 1-hour follow-up scan and an additional $12 \%$ had larger hemorrhages at the $20-\mathrm{h}$ scan. The change in hematoma volume was associated with clinical deterioration [49]. HE has also been demonstrated in smaller series for as many as 5 and 6 days [52]. Even though early HE and its associated neurological worsening have been clearly demonstrated, the risk factors associated with HE have not been defined in large population studies [47, 49-51]. Current efforts are focused on identification of patients who will develop hematoma expansion with clinical, radiographic, or molecular markers. Certain biomarkers have been seen to be elevated in ICH, such as interleukin-6 and cellular fibronectin [53]. As previously mentioned, APOE $\varepsilon 2$ may also be associated with subsequent hematoma expansion [25]. In addition, treatment efforts are directed at halting hematoma expansion. Because HE occurs in approximately a third of ICH patients, and because it is independently associated with worsening of 
clinical status and outcome [54], HE represents a promising acute treatment target.

\section{Hemostatic Agent}

Recombinant factor VIIa (rFVIIa) has been studied to limit HE. After initial safety studies, a proof-of-concept trial was performed to observe the effect of rFVIIa on HE [55]. This study randomized patients who presented within 3 hours of symptom onset to either a single dose of $40 \mu \mathrm{g} / \mathrm{kg}, 80 \mu \mathrm{g} / \mathrm{kg}$, or $160 \mu \mathrm{g} / \mathrm{kg} \mathrm{rFVIIa}$, or placebo within $1 \mathrm{~h}$ of baseline CT. Mean percent increase and mean absolute change in ICH volume was significantly lower in the $160 \mu \mathrm{g} / \mathrm{kg}$ group than in the placebo group. Thromboembolic events occurred in $2.1 \%$ in the placebo group and $6.9 \%$ in the treated group. The results of this proof of concept trial lead to a confirmatory efficacy study with similar enrollment criteria, except the exclusion for a history of thromboembolic disease was removed. The results were disappointingly neutral. There was no difference in the primary outcome measure of death or severe disability (modified Rankin Scale score (mRS), 5 or 6) in patients who received placebo compared with those who received rFVIIa. There was, however, a dose-related decrease in HE accompanied by a dose-related increase in adverse events in patients who received rFVIIa. The percent of change in ICH volume at $24 \mathrm{~h}$ was $28 \%$ in those receiving placebo, and $18 \%$ and $11 \%$ in the $20 \mu \mathrm{g} / \mathrm{kg}$ and $80 \mu \mathrm{g} / \mathrm{kg}$ groups, respectively. The percent of arterial thromboembolic events was $10 \%$ in the $80 \mu \mathrm{g} / \mathrm{kg}$ group and $5 \%$ in the placebo group, without any difference in venous thromboembolism [56].

Several explanations have been postulated as to why the clear radiographic benefit of rFVIIa did not translate to improved clinical outcome (e.g., time to treatment, older age, better-than-expected outcomes in the placebo group, and an increase in arterial thromboembolic events) [56]. However, the most salient explanation has to do with patient selection. Because there are only approximately one-third of patients who are expected to have acute HE, the treatment effect of rFVIIa may have been dampened by the enrollment of patients who would not have had significant enlargement, and thus would not be expected to benefit from this treatment. One way to improve patient selection for hemostatic treatment is to predict which patients are more likely to have significant HE and target that group for treatment. A radiographic marker of HE, the spot sign, has been retrospectively evaluated, and its presence has been found to be correlated with the risk for HE [57-59]. The spot sign represents a small focus or larger area of contrast extravasation within the hematoma on CT angiography, most often seen on source images [57]. In addition, contrast extravasation, seen on routine head CT after CT angiography, as pooling of contrast within the hematoma, may be a more sensitive predictor of ICH growth with a better negative predictive value than the spot sign [60]. A new study, Spot Sign for Predicting and Treating ICH Growth (STOP-IT) [61], is planned to evaluate the usefulness of rFVIIa in individuals with an ICH spot sign.

\section{Blood Pressure}

One of the most common clinical presentations in the acute setting of ICH is elevated blood pressure. In the acute period of ICH, elevated blood pressure has been correlated with a poor outcome in several studies. A meta-analysis performed by Willmot et al. [62] included 32 studies involving 10,892 ischemic and hemorrhagic stroke patients, which found that death was significantly associated with an elevated mean arterial blood pressure (odds ratio [OR], $1.61 ; 95 \%$ confidence interval [CI] 1.12-2.31) and high diastolic blood pressure (OR, 1.71; 95\% CI 1.33-2.48). Combined death or dependency was associated with high systolic blood pressure (OR, 2.69; 95\% CI 1.13-6.40) and diastolic blood pressure (OR, 4.68; 95\% CI 1.87-11.70) in ICH. In another large recent Chinese trial that included a total of 1760 hemorrhagic stroke patients, it was found that the diastolic blood pressures were significantly and positively associated with odds of death or disability in acute hemorrhagic stroke. Compared to those with a systolic blood pressure less than $140 \mathrm{mmHg}$, multiple-adjusted odds ratio (95\% confidence interval) of death/disability was $1.38(0.96$, 1.99), 1.42 (1.00, 2.03), $1.84(1.28,2.64)$, and 1.91 (1.35, 2.70) among participants with systolic blood pressure of 140 to 159,160 to 179,180 to 199 , and at least $200 \mathrm{mmHg}$, respectively ( $p<0.0001$ for linear trend) [63]. One interpretation of this data would be that rapid reduction in blood pressure might decrease the chances of poor outcome; however, this evidence does not demonstrate causality or reveal whether or not the aggressive management of elevated blood pressure in the acute setting will improve clinical outcome.

The association between elevated blood pressure and increased risk for HE is not clear, as some studies have not been able to define a relationship between the 2 [47, 49], and others have shown that there may be an associated risk for HE [64]. Controversy exists regarding the acute treatment of hypertension in ICH because of the possible expansion of what was once believed to be ischemic penumbra surrounding the hematoma. However, more recent positron emission tomographic evidence has shown that the area surrounding the hematoma is more likely to be due to metabolic suppression and not ischemia, and it is not affected by blood pressure reduction [65].

The results of 2 pilot trials designed to assess the feasibility and safety of rapid blood pressure reduction in the acute setting of an ICH have been reported. The Intensive Blood Pressure Reduction in Acute Cerebral 
Hemorrhage Trial (INTERACT) randomized patients with acute spontaneous ICH within 6 hours of symptom onset, elevated systolic blood pressure (150-220 $\mathrm{mmHg}$ ), and no definite indication or contraindication to treatment to early intensive lowering of blood pressure (target systolic blood pressure, $140 \mathrm{mmHg} ; \mathrm{n}=203$ ) or standard guideline-based management of blood pressure (target systolic blood pressure, $180 \mathrm{mmHg} ; \mathrm{n}=201$ ). The authors concluded that early intensive blood pressure lowering treatment is clinically feasible, well tolerated, and seems to reduce hematoma growth in ICH [66]. The second phase of INTERACT, known as The Second Intensive Blood Pressure Reduction in Acute Cerebral Hemorrhage Trial (INTERACT 2) [67], is now underway and plans to enroll 2800 patients who will be randomized to reducing their systolic blood pressure to less than $140 \mathrm{mmHg}$ within $1 \mathrm{~h}$ or less than $180 \mathrm{mmHg}$. The primary outcome measure of this study is death and dependency at 3 months. One drawback of INTERACT is the majority of patients are of Chinese descent, which raises the question as to whether or not the results are generalizable.

The Antihypertensive Treatment in Acute Cerebral Hemorrhage (ATACH) trial was a nonrandomized, openlabel study, which also assessed the feasibility and safety of acute blood pressure reduction in ICH. This pilot study differed from INTERACT in that there were 3 different groups of blood pressure goals and all patients were treated with nicardipine unlike INTERACT, which allowed the use of any available local intravenous anti-hypertensive agent. The ATACH trial consisted of 3 cohorts with systolic blood pressure ranging from 170 to $200 \mathrm{mmHg}$ in the first cohort of patients; 140 to $170 \mathrm{mmHg}$ in the second cohort; and 110 to $140 \mathrm{mmHg}$ in the third cohort. Cohorts 2 and 3 had a trend toward worse outcome and more numerical deaths than cohort 1; however, the study was not powered to show an effect on death and disability. Consistent with findings from INTERACT, the study demonstrated that rapid blood pressure reduction was safe and feasible [68]. The ATACHII trial, which is the next phase of ATACH, is a phase 3, randomized efficacy study designed to evaluate the therapeutic benefit of intensive acute blood pressure treatment (systolic blood pressure $\leq 140 \mathrm{mmHg}$ ) with intravenous nicardipine within $3 \mathrm{~h}$ of symptom onset compared with standard care (systolic blood pressure $\leq 180 \mathrm{mmHg}$ ). The primary outcome measure is death or disability (mRS, 4-6) at day 90 . The study began in 2010 and plans to enroll 1280 patients [69].

\section{Secondary Processes}

The secondary pathophysiological processes have been implicated as causes of ongoing, injury including ischemia surrounding the hematoma, the development of cerebral edema [70], activation of apoptotic processes [71], toxic effects of components of the hematoma [72], and intraventricular extension of the primary hemorrhage [73]. The pathogenesis of symptoms and their subsequent recovery are also associated with hematoma size and location. Smaller hemorrhages may dissect along white matter tracts, which actually splits the fibers from each other instead of destroying them. If the hematoma takes this path, then there may be a higher likelihood of restoration of neurological function once the blood is resorbed. However, large clots, especially those with a cortical location involving the grey matter may result in a more sustained deficit. Hematomas greater than $5 \mathrm{~cm}$ in diameter have a larger risk of expansion associated with local irritation and tearing of tissue at its edge [74].

In addition to the hematoma, the associated edema may also contribute to the initial neurological deficit, subsequent decline, or death. The edema related to ICH has been cited as a reason for neurological deterioration after the first 24 to $48 \mathrm{~h}$ from the onset of symptoms [70], and it has, to a lesser degree, also been implicated with deterioration as late as 3 weeks [65]. The edema has been demonstrated to be predominately vasogenic with a cytotoxic component. The vasogenic edema is a consequence of blood brain barrier (BBB) breakdown. In the normal brain, the BBB prevents the flow of water into the brain due to hydrostatic pressure gradients. However, when the BBB is disrupted as occurs in $\mathrm{ICH}$, the imbalance in hydrostatic forces result in the entry of an exudative proteinaceous fluid onto the brain parenchyma [74]. The disruption in the BBB is likely a consequence of an inflammatory cascade with resultant expression of specific cytokines and other markers of inflammation. The presence of red blood cells and their subsequent lysis and release of oxyhemoglobin may contribute to the leakage of the BBB [75]. The hemorrhage itself also induces the production of thrombin and the overexpression of matrix metalloproteinases. Thrombin has been demonstrated to be an important factor in the modulation of BBB breakdown [76]. Thrombin may be a major mediator of ICH-induced tumor necrosis factor- $\alpha$ production and an increase of perihematomal tumor necrosis factor- $\alpha$ levels contributes to brain edema formation after ICH [76]. Matrix metalloproteinases also promote BBB disruption [77, 78] and have been associated with increased edema volume [78] via extracellular matrix proteolysis, basal lamina destruction, and the degradation of c-fibronectin [79].

Cellular necrosis likely occurs at the core of the hemorrhage; however, apoptosis has been observed in the perihematomal region [71]. The apoptotic pathway in ICH may involve nuclear factor-kappa B (NF-kB), which is a ubiquitous transcription factor that, when activated, translocates to the nucleus and binds to DNA. NF- $k \mathrm{~B}$ is associated with apoptotic cell death and has been reported 
in the role of cell death after experimental ICH in rats [80]. In experimental models, peak apoptosis levels in the perihematomal area have been observed to occur on day 3 [81]. In addition to NF- $\mathrm{KB}$, the activation of caspase 3 and the role of thrombin have also been demonstrated in perihematomal apoptosis [81].

The secondary injury caused by ICH is complex, but it offers multiple treatment targets. Currently, researchers are still elucidating the sequence of events of downstream processes and how each interacts. Each of these targets requires important preclinical data to inform clinical trials with respect to the optimal time frame for treatment, treatment duration, dose, and so forth. Without this important foundation, cytoprotective trials in ICH run the risk of falling into the same situation that has plagued ischemic stroke (i.e, namely, difficulty in translation of improved clinical outcomes from bench to bedside).

\section{Clinical Trials Targeting Secondary Injury}

\section{Surgical Hematoma Clearance}

Supratentorial surgical evacuation of an ICH has been a strongly debated topic in regards to its efficacy in producing good clinical outcomes. The current available evidence of surgical evacuation is largely based on the randomized prospective International Surgical Treatment of Intracerebral Hemorrhage (STICH) study [82]. Other available evidence includes a meta-analysis of 3 previously reported trials and case series [83]. The broad conclusion from these studies was that there was no difference in mortality or outcome between the groups randomized to surgical evacuation of the hematoma compared to medical management. Criticisms for STICH are that there was often crossover from the medical arm to the surgical arm and that many patients were taken to surgery beyond the predefined $24 \mathrm{~h}$, which makes the results difficult to interpret. Interestingly, a subgroup analysis of patients with hematomas extending within $1 \mathrm{~cm}$ from the cortical surface and patients with a Glasgow coma scale (GCS) of 9 to 12 showed a trend toward a favorable outcome in patients who were randomized to surgery within 96 hours compared to medical management. However, neither of these results reached statistical significance [82]. This information has been used to form a follow-up trial called the Surgical Trial in Lobar ICH (STICH-II) [84], which is studying the possible benefit of surgical evacuation of cortical hemorrhages.

Other less invasive approaches to evacuation of the ICH are also being investigated. A small phase 2 study conducted by Vespa et al. [85] has shown that hematoma aspiration via a catheter is safe in conjunction with the use of thrombolytics to remove unclotted blood. This data has led to the development of the Minimally Invasive Stereotactic
Surgery + [recombinant tissue plasminogen activator] rt-PA for ICH Evacuation (MISTIE) trial [86], which is comparing standard medical management to hematoma evacuation using a stereotactic catheter plus a thrombolytic-based approach.

\section{Intraventricular Hemorrhage}

One-third of patients with spontaneous ICH due to chronic arterial hypertension or small arteriolar degeneration and rupture have an associated intraventricular hemorrhage (IVH). The most common locations of an ICH associated with an IVH are thalamic, putaminal head, or caudate, resulting in spread to the lateral or third ventricles [74]. There is an established relationship between IVH with larger ICHs, midline shift, and increased morbidity and mortality [73]. An important consequence of IVH is hydrocephalus, which can develop acutely or gradually with time due to scarring of the arachnoid granulations from blood products. The clearance of the ventricular blood from the cerebrospinal fluid (CSF) occurs via several mechanisms. First, hemolysis occurs and begins within hours, reaching a plateau at 2 to 10 days after the IVH, depending on the size of the hemorrhage. Next is phagocytosis by macrophages, which occurs both in the leptomeninges irritated by blood and in the arachnoid granulations engorged with erythrocytes [87]. The breakdown of the actual clot within the ventricles is mediated by plasmin in the CSF. Plasmin is present in the CSF similar to serum in its precursor form plasminogen. As with serum, plasmin in the CSF is converted to its active form by tissueplasminogen activator, which is released from the endothelium of the ependyma. The level of fibrinolytic activity is lower than plasma, but it is proportional to the clot burden in the ventricles and balanced by inhibitors released by irritated leptomeninges [87, 88]. Currently the only available treatment for IVH with hydrocephalus is the placement of an external ventricular drain; however, the external ventricular drain can become difficult to manage because of frequent obstruction from a thrombus resulting in interrupted drainage and increased intracranial pressure (ICP). An ongoing, multicenter study is attempting to evaluate a solution to this problem. The Clot Lysis: Evaluating Accelerated Resolution of Intraventricular Hemorrhage (CLEAR IVH) trial is evaluating the safety and efficacy of intraventricular recombinant tissue plasminogen activator in clearing intraventricular blood and facilitating its drainage to help improve outcome in patients with IVH [89].

\section{Pharmacologic Hematoma Clearance}

The deposited blood causes local tissue deformation and elicits an inflammatory response after ICH. Although the inflammation involves most of the brain's cell types, the 
brain's resident phagocytes, microglia, are the most prominent, and become activated within minutes after ICH $[90,91]$. The activated microglia release chemotactic factors, which then recruit hematogenous phagocytes to the hemorrhagic area. These activated phagocytes engulf the deposited blood, damaged and dead tissue, and then provide a nurturing environment for local tissue reconstruction. It has been proposed that phagocytosis not only removes debris from the tissue, but also provides protection from local damage resulting from the release of toxic or proinflammatory contents [92, 93]. Microglia may also play a role in establishing trophic support involved in neuronal sprouting [94] and forming new vessels, which could then be used for removal of brain tissue debris after injury [95]. Ultimately, it is the inflammation (phagocytosis) that leads to the restoration of tissue structure and function [96, 97]. Although inflammation is triggered primarily to remove the blood and other debris left by the hemorrhage, the byproducts of this response are cytotoxic and lead to further tissue damage, blood brain barrier disruption, and edema [72, 97, 98].

One way to reduce the production of blood degradation products is hematoma evacuation. This has been the impetus for trials of hematoma evacuation, which, unfortunately, have not demonstrated benefit. However, few studies have focused on the natural history of hematoma absorption [75, 99-101]. With current clinical efforts focused on ICH treatment in the acute setting, a treatment that could enhance hematoma resolution, thus limiting secondary injury, would be a natural adjunctive treatment to acute therapy for this disease.

Another way to decrease the clot burden is to take advantage of the body's own mechanism of clot removal (i.e., phagocytosis). Pre-clinical work in our laboratory demonstrates that peroxisome proliferators-activated receptor-gamma (PPAR- $\gamma$ ) agonists promote hematoma resolution, decrease neuronal damage, and improve functional recovery in a mouse model of ICH [102]. We have also demonstrated that PPAR- $\gamma$ agonists in vitro reduce production of proinflammatory mediators and free radicals produced during phagocytosis. Finally, PPAR- $\gamma$ agonists in vivo demonstrate the ability to protect other brain cells from the secondary injury induced in a mouse model of ICH [102]. These data suggest that PPAR- $\gamma$ in macrophages acts as an important factor in promoting hematoma absorption and protecting other brain cells from ICH-induced damage and may represent a promising therapeutic target in the management of ICH [103]. Clinically relevant PPAR $-\gamma$ agonists include rosiglitazone and pioglitazone, which are approved by the Food and Drug Administration for glycemic control in type 2 diabetes mellitus.

The Safety of Pioglitazone for Hematoma Resolution In ICH (SHRINC) study is a prospective, randomized, placebo-controlled, dose-escalation safety trial in which patients with spontaneous ICH are randomly allocated to placebo or to treatment with escalating doses of pioglitazone followed by a maintenance dose for the duration of treatment. Functional outcomes are evaluated at 3 and 6 months. There is a planned sample size of 80 patients and enrollment is $50 \%$ complete [104].

\section{Edema: Celecoxib}

In the collagenase model of $\mathrm{ICH}$, celecoxib demonstrated a time-dependent improvement in sensorimotor function that was persistent for as much as 4 weeks after the injury. In addition, celecoxib reduced infiltration of inflammatory cells, brain edema, and subsequent perihematomal cell death [105]. The authors postulate that this effect may be due to a decrease in prostaglandin E2, because prostaglandin E2 was markedly reduced in the celecoxib-treated animals.

A small, retrospective study investigated the efficacy and safety of celecoxib treatment in patients with ICH compared to a similar control group. Initial and followup hematoma and edema volumes of 17 patients with ICH who received celecoxib for $\geq 7$ days were compared with a control group. Celecoxib treatment was significantly associated with a reduction in the volumes of edema on follow-up brain imaging compared with the volumes in the control group $(30.2 \pm 17.7$ vs $55.5 \pm 40.6 \mathrm{~mL} ; p=0.027)$. There was no difference in the number of adverse events between the 2 groups. These results suggested that celecoxib may be safe and efficacious in patients with primary ICH [106].

To further evaluate these promising findings, a multicenter trial, named the Prospective, Randomized, Comparative Open with Blinded Endpoints (PROBE) trial, was developed to assess the safety and efficacy of administration of celecoxib in patients with ICH. The Administration of Celecoxib for Treatment of ICH: A Pilot Study (ACE-ICH) began enrollment in 2007 and was completed in 2009. The primary outcome measure was the change in perihematomal edema for a period of time. Patients were randomized to receive either celecoxib (400 mg twice daily) for a duration of 14 days or a placebo within $24 \mathrm{~h}$ of symptom onset. The primary outcome measure was change in volume of perihematomal edema for a period of time. Secondary outcome measures included neurologic function and the incidence of adverse events. The study was recently completed and the results will be available soon (personal communication, Dr. Roh).

\section{Blood, Brain, Barrier Disruption: Albumin}

It is difficult to ignore the similarities in mechanism of injury between acute ischemic stroke and $\mathrm{ICH}$, namely, blood brain barrier disruption [107], edema, oxidative injury, inflammation, excitotoxicity, and apoptosis [108]. Thus, it 
seems intuitive that treatments that provide neuroprotection in ischemic stroke might also do the same in ICH. Albumin has demonstrated neuroprotection in preclinical models of cerebral ischemia and researchers have attempted to extend the application of albumin to ICH as well.

In preclinical studies, albumin therapy administered 60 minutes after ICH improved neurological function as early as $2 \mathrm{~h}$ after treatment and was maintained through day 3 in a cortical model of ICH. Albumin also improved BBB integrity [107]. This group demonstrated similar findings in a subcortical model of ICH with albumin administered 90 minutes after ICH. In both studies, neither hematoma volume nor brain swelling were affected by albumin [109].

The Albumin for ICH Intervention (ACHIEVE) study is a phase 2, placebo-controlled trial evaluating the effects of albumin in patients with ICH within $24 \mathrm{~h}$ of symptom onset. The primary outcome measure is the frequency and severity of blood brain barrier disruption based on a magnetic resonance image with and without contrast. Secondary outcome measures included safety endpoints. The study began in 2009 with a planned enrollment of 40 patients and an estimated completion in 2012 [110].

\section{Red Blood Cell Breakdown and Toxic Breakdown Products: Free Radical Scavengers}

Several nitrone free radical-trapping agents (i.e., spin-trap agents) have demonstrated neuroprotection in rodent models of both transient and permanent focal ischemia [111, 112]. NXY-059 (disodium 4-[(tert-butylimino) methyl] benzene-1,3-disulfonate $\mathrm{N}$-oxide) is a novel nitrone-based compound that has free radical-trapping properties.

The Cerebral Hemorrhage and NXY Treatment (CHANT) study was a randomized, placebo-controlled trial that evaluated the safety and tolerability of NXY-059 (a free radical trapping agent) in patients with ICH within $6 \mathrm{~h}$ of symptom onset. At the time, NXY-059 was also being evaluated for potential treatment in patients with acute ischemic stroke. The intent of CHANT in ICH patients was to facilitate prompt administration of the treatment, potentially before neuroimaging. A total of 607 patients were randomized within $6 \mathrm{~h}$ of acute ICH (300 with NXY-059 vs 303 with a placebo). Mortality was similar in both groups: $20.3 \%$ for NXY-059 and $19.8 \%$ for placebotreated patients. The proportion of patients who experienced an adverse event was the same for both groups, whereas for serious adverse events the proportion was slightly higher in the NXY-059 group; however, no pattern emerged to indicate a safety concern. There were no differences in 3-month function, disability, or neurological deficit scores. Based on this study, the authors concluded that NXY-059 given within 6 hours of acute ICH has a good safety and tolerability profile, with no adverse effect on important clinical outcomes [113]. It is unclear if there are plans to further evaluate this treatment.

\section{Chelating Agent: Deferoxamine}

At this time, deferoxamine (DFO) is probably the most thoroughly evaluated treatment in animal models of ICH, in terms of informing potential translational clinical efforts. Currently, DFO is further along in complying with the updated Stroke Therapy Academic Industry Roundtable (STAIR) recommendations [114] than most other treatments being evaluated in the preclinical setting. DFO has been studied in both the collagenase [115] and autologous whole blood models of ICH, as well as in both rodent and pig models [116] of ICH. DFO has been studied in both young and aged rodents [117] and has been shown to cross the BBB. The most recent studies evaluating DFO have addressed optimal doses and time windows for treatment [118]. The preclinical work with DFO consistently demonstrates decreased iron accumulation and improved neurologic function $[115,118,119]$.

The Dose Finding and Safety Study of Deferoxamine in Patients with ICH (DFO in ICH) Study was a phase I open label study evaluating the safety and tolerability of varying doses of DFO to determine a maximum tolerated dose to be adopted in an efficacy trial in patients with ICH [120]. The study began in 2008 and was recently completed. The results were recently reported and demonstrated that repeated daily infusions of DFO in doses up to $62 \mathrm{mg} / \mathrm{kg} / \mathrm{day}$ in patients with acute spontaneous ICH are feasible, well tolerated, and are not associated with an increase in serious adverse effects or mortality. Phase 2 evaluation of DFO is currently in the planning stages [121].

\section{Anti-Inflammatory Agents: Statins}

Despite the association of low cholesterol levels $[122,123]$ and the use of a statin with ICH [124], there is a growing interest in the pleiotropic effects of the 3-hydroxy-3methylglutaryl coenzyme A reductase inhibitors (i.e., statins). The promising neuroprotective benefit of the statins in acute ischemic stroke [125-127] has led to a similar evaluation in ICH models. In the autologous whole blood model, atorvastatin at $2 \mathrm{mg} / \mathrm{kg}$ was beneficial, and higher doses did not improve outcome or reduce the extent of injury [128]. Atorvastatin reduced perihematoma cell death via an anti-inflammatory mechanism and this was also associated with sensorimotor recovery [129].

Both simvastatin and atorvastatin treatment for 1 week after ICH significantly improved neurological outcome and reduced hematoma volume and tissue loss at 4 weeks. The neurological improvement became apparent at 1 week and was consistent throughout 4 weeks. In addition, the statins increased cell proliferation and differentiation suggesting that enhanced neuroplasticity may be the mechanism for improved function $[128,130]$. 
In the collagenase ICH model, atorvastatin exerted protection against $\mathrm{ICH}$ via modulation of inflammation. Taken together, these results suggest that statins may shift the balance of inflammation, such that the general milieu favors enhanced neuroplasticity, and thus the potential for improved recovery. The possible mechanisms of neuroprotection may include improved endothelial function, increased endothelial nitric oxide synthase expression [129], antioxidant effects, conferred resistance to N-methyl-D-aspartate excitotoxicity [131], promotion of neovascularization, and anti-inflammatory properties.

Although encouraging, the amount of preclinical data on the effect of statins in ICH is limited and some findings are not consistent. For example, 2 groups have demonstrated an effect of statins on hematoma volume $[128,130]$, whereas another group has not [129]. Thus far, preclinical studies demonstrate an effect on hematoma volume, brain atrophy, hemispheric water content, and neurologic function; however, clinical evaluation of the statins has been limited to a demonstration of associations between statin use and mortality, and perihematomal edema. Retrospective evaluation in a cohort of ICH patients demonstrated that prior statin use was found to be associated with decreased mortality with a greater than 12 -fold odds of survival $(p=0.05)$. The same patient population also demonstrated an association between statin use prior to $\mathrm{ICH}$ and decreased absolute and relative perihematomal edema at presentation [132]. There is a report of 1 small, prospective cohort of 18 patients treated with rosuvastatin compared to 57 historical controls [133]. The mortality rate during hospitalization was $5.6 \%$ in the statin group and $15.8 \%$ in the control group with an adjusted hazard ratio of 0.20 (95\% CI $0.02-1.67)$, also suggesting that the use of statins during the acute phase of ICH may be associated with improved mortality.

The Simvastatin for ICH Study is a phase 2, randomized, blinded, placebo-controlled, efficacy study evaluating treatment with $80 \mathrm{mg}$ of simvastatin or a placebo for 14 days on perihematomal edema. Secondary outcome measures include mortality at 30 days and functional outcome at 90 days. There is a planned sample size of 90 patients. The study began in 2008 and completion is expected in 2011 [134].

\section{Other Treatment Considerations}

Management of ICH in Patients on Concurrent Antithrombotic Therapy

The outcomes in individuals on antiplatelet therapy with an ICH are variable according to the available literature [135139]. The best evidence includes a post-hoc analysis of the placebo arm of the CHANT study, which included 70 patients who were on antiplatelet therapy at onset of their $\mathrm{ICH}$. The authors found that antiplatelet medications at ICH onset had no association with the volume of ICH at presentation, growth of ICH at $72 \mathrm{~h}$, initial edema volume, or edema growth [139]. However, a meta-analysis by Thomson et al. [140] found that the use of antiplatelets at the time of ICH compared to no use was independently associated with increased mortality, but not with poor functional outcome. A retrospective analysis of patients who were on antiplatelet therapy prior to their ICH revealed they had an increased rate of death, and platelet transfusion did not prevent death or improve outcome [141]. Based on this limited information, some clinicians favor the use of platelet transfusion when patients present with an ICH and are on antiplatelet agents. There is data that reports an association with increased mortality when ICH patients receive blood products [142] and more information is needed to determine whether platelet transfusion should be a consideration for patients on antiplatelet therapy who present with ICH.

The Platelet Transfusion in Acute Intracerebral Hemorrhage trial was designed to help address this issue. This is an open-label study that will evaluate whether the use of antiplatelet agents results in an increased risk of hematoma enlargement after acute ICH. In addition, the study will evaluate the safety and efficacy of platelet transfusion for prevention of hematoma growth in patients who develop acute ICH while taking an antiplatelet agent. The primary outcome measure is hematoma growth within $24 \mathrm{~h}$. Secondary outcome measures include Glasgow outcome, cardiovascular death occurring within the treatment period, death due to any cause occurring within the treatment, acute myocardial infarction, and venous thromboembolism at 90 days [143]. In addition, the Improving Platelet Activity for Cerebral Hemorrhage Treatment - DDAVP Proof of Concept (IMPACT) study is a phase 2, open label study in which patients who have spontaneous ICH are on aspirin or have a laboratory marker indicating the use of antiplatelet medication will receive a DDAVP injection (desmopressin acetate, $0.4 \mathrm{mcg} / \mathrm{kg}$ ) to determine whether DDAVP improves platelet activity from baseline to 60 minutes after treatment start. The primary outcome measure is change in platelet activity, measured in seconds on platelet function assay, from pre to post-treatment [144]. Both of these studies will begin to fill a gap in the existing literature regarding the use of antiplatelets and blood products in the setting of $\mathrm{ICH}$.

ICH associated with anticoagulation due to vitamin $\mathrm{K}$ antagonists is increasing in incidence [12]. Recent new anticoagulants, such as oral direct thrombin inhibitors, will likely contribute to the challenges of anticoagulationrelated hemorrhages with more widespread use [145]. 
Individuals on warfarin with an international normalized ratio $>3$ have an increased risk for having larger hematomas [146]. To be able to potentially curtail the hematoma growth of different agents, including vitamin $\mathrm{K}$ (either with or without fresh frozen plasma), factor VII, and prothrombin complex concentrates have been used. There is no randomized controlled data to suggest that 1 agent is better than the other; however, the most recent guidelines for the management of ICH recommend against routine use of rFVIIa for warfarin reversal [147]. The dilemma of which agent to use is increased with oral direct thrombin inhibitors, such as dabigatran, and factor Xa inhibitors, such as rivaroxaban, as there are no proven therapies to reverse their anticoagulant effect.

Other Treatment Modalities Additional treatments that are being evaluated for ICH treatment include hypothermia [148, 149], stem cells, minocycline [98, 150], and rehabilitation interventions [151-153]. The latter treatment modalities are currently in the early stages of preclinical development. Regarding hypothermia, there is a planned clinical trial to evaluate the efficacy of hypothermia as acute treatment of patients with primary ICH. As of now, the trial is not yet open for enrollment. The primary outcome measures are neurologic function at 3 and 6 months. The trial will include patients with primary ICH who present with $\mathrm{GCS} \leq 8$ and an ICH score of 2 to 4 . Induction of hypothermia will begin within $6 \mathrm{~h}$ of symptom onset. Patients will be cooled to a core temperature of $34^{\circ} \mathrm{C}$ during $24 \mathrm{~h}$ with surface cooling [154].

\section{Conclusions}

Current challenges in developing treatment for ICH are the same challenges that are faced with acute ischemic stroke; these include having a solid preclinical foundation to inform clinical trials, choosing the appropriate clinical and surrogate outcome measures, and identifying the appropriate patient population. Given the challenges in addressing the heterogeneity of the patient population and the history of clinical trials in ischemic stroke, it may be necessary to consider changes in clinical trial design, such as adaptive designs for randomization, dose escalation, and outcome analyses.

To have an impact on this devastating disease, multiple treatment targets must be identified. The history of clinical trials in acute ischemic stroke tells us that the future of stroke treatment, both ischemic and hemorrhagic, will be multi-faceted with combined therapies aimed at both the primary and secondary injuries caused by disruption of the vasculature. In $\mathrm{ICH}$, the most promising clinical trial was with the hemostatic agent, rFVIIa, which had a 4-h treatment window. Because a large number of patients with ICH may not be eligible for acute treatment options, given the well-known limitations in presentation to the emergency department, adjunctive therapy to more acute treatments are also being targeted. In addition, clinical and radiographic data are being used to identify the patient population most likely to respond to specific therapies. This multifaceted approach to the treatment of ICH lends hope for a treatment to be developed soon. The ideal treatment strategy will be one that is practical and applicable for both specialized stroke centers and community hospitals.

Acknowledgment Full conflict of interest disclosure is available in the electronic supplementary material for this article.

\section{References}

1. Cheung RT. Update on medical and surgical management of intracerebral hemorrhage. Rev Recent Clin Trials 2007;2:174-181.

2. Broderick JP, Phillips SJ, Whisnant JP, O'Fallon WM, Bergstralh EJ. Incidence rates of stroke in the eighties: the end of the decline in stroke? Stroke 1989;20:577-582.

3. Giroud M, Gras P, Chadan N, et al. Cerebral haemorrhage in a French prospective population study. J Neurol Neurosurg Psychiatry 1991;54:595-598.

4. Broderick JP, Brott T, Tomsick T, Huster G, Miller R. The risk of subarachnoid and intracerebral hemorrhages in blacks as compared with whites. N Engl J Med 1992;326:733-736.

5. Suzuki K, Kutsuzawa T, Takita K, et al. Clinico-epidemiologic study of stroke in Akita, Japan. Stroke 1987;18:402-406.

6. Labovitz DL, Halim A, Boden-Albala B, Hauser WA, Sacco RL. The incidence of deep and lobar intracerebral hemorrhage in whites, blacks, and Hispanics. Neurology 2005;65:518-522.

7. Bamford J, Sandercock P, Dennis M, Burn J, Warlow C. A prospective study of acute cerebrovascular disease in the community: the Oxfordshire Community Stroke Project-198186. 2. Incidence, case fatality rates and overall outcome at one year of cerebral infarction, primary intracerebral and subarachnoid haemorrhage. J Neurol Neurosurg Psychiatry 1990;53:16-22.

8. Rosamond W, Flegal K, Friday G, et al. Heart disease and stroke statistics - 2007 update: a report from the American Heart Association Statistics Committee and Stroke Statistics Subcommittee. Circulation 2007;115:e69-e171.

9. Diringer MN, Edwards DF. Admission to a neurologic/neurosurgical intensive care unit is associated with reduced mortality rate after intracerebral hemorrhage. Crit Care Med 2001;29:635-640.

10. Varelas PN, Schultz L, Conti M, Spanaki M, Genarrelli T, Hacein-Bey L. The impact of a neuro-intensivist on patients with stroke admitted to a neurosciences intensive care unit. Neurocrit Care 2008;9:293-299.

11. Xi G, Keep RF, Hoff JT. Mechanisms of brain injury after intracerebral haemorrhage. Lancet Neurol 2006;5:53-63.

12. Flaherty ML, Kissela B, Woo D, et al. The increasing incidence of anticoagulant-associated intracerebral hemorrhage. Neurology 2007;68:116-121.

13. Rosand J, Eckman MH, Knudsen KA, Singer DE, Greenberg SM. The effect of warfarin and intensity of anticoagulation on outcome of intracerebral hemorrhage. Arch Intern Med 2004;164:880-884.

14. Hart RG, Tonarelli SB, Pearce LA. Avoiding central nervous system bleeding during antithrombotic therapy: recent data and ideas. Stroke 2005;36:1588-1593. 
15. Shen AY, Yao JF, Brar SS, Jorgensen MB, Chen W. Racial/ethnic differences in the risk of intracranial hemorrhage among patients with atrial fibrillation. J Am Coll Cardiol 2007;50:309-315.

16. O'Donnell HC, Rosand J, Knudsen KA, et al. Apolipoprotein E genotype and the risk of recurrent lobar intracerebral hemorrhage. N Engl J Med 2000;342:240-245.

17. Greenberg SM, Vonsattel JP. Diagnosis of cerebral amyloid angiopathy. Sensitivity and specificity of cortical biopsy. Stroke 1997;28:1418-1422.

18. Vinters HV. Cerebral amyloid angiopathy. A critical review. Stroke 1987;18:311-324.

19. Vonsattel JP, Myers RH, Hedley-Whyte ET, Ropper AH, Bird ED, Richardson EP, Jr. Cerebral amyloid angiopathy without and with cerebral hemorrhages: a comparative histological study. Ann Neurol 1991;30:637-649.

20. Greenberg SM, Briggs ME, Hyman BT, et al. Apolipoprotein E epsilon 4 is associated with the presence and earlier onset of hemorrhage in cerebral amyloid angiopathy. Stroke 1996;27:13331337

21. Nicoll JA, Burnett C, Love S, et al. High frequency of apolipoprotein $\mathrm{E}$ epsilon 2 allele in hemorrhage due to cerebral amyloid angiopathy. Ann Neurol 1997;41:716-721.

22. Yamada M, Itoh Y, Suematsu N, Matsushita M, Otomo E. Lack of an association between apolipoprotein $\mathrm{E}$ epsilon 4 and cerebral amyloid angiopathy in elderly Japanese. Ann Neurol 1996;39:683-684.

23. Greenberg SM, Vonsattel JP, Segal AZ, et al. Association of apolipoprotein E epsilon2 and vasculopathy in cerebral amyloid angiopathy. Neurology 1998;50:961-965.

24. Biffi A, Sonni A, Anderson CD, et al. Variants at APOE influence risk of deep and lobar intracerebral hemorrhage. Ann Neurol 2010;68:934-943.

25. Biffi A, Kissela B, Ayres AM, et al. International Stroke Conference Oral Presentations: APOE Genotype Predicts Hematoma Volume and Expansion in Intracerebral Hemorrhage. Stroke 2011;42:e102.

26. Schmechel DE, Saunders AM, Strittmatter WJ, et al. Increased amyloid beta-peptide deposition in cerebral cortex as a consequence of apolipoprotein E genotype in late-onset Alzheimer disease. Proc Natl Acad Sci U S A 1993;90:9649-9653.

27. Gervais F, Chalifour R, Garceau D, et al. Glycosaminoglycan mimetics: a therapeutic approach to cerebral amyloid angiopathy. Amyloid 2001;8 Suppl 1:28-35.

28. Greenberg SM. Cerebril ${ }^{\mathrm{TM}}$ in Patients With Lobar Hemorrhage Related to Cerebral Amyloid Angiopathy. [Cited 2010 December 21]; available from: http://clinicaltrials.gov/ct2/show/NCT00056238; NLM Identifier: NCT00056238. Accessed December 21.

29. Gould DB, Phalan FC, van Mil SE, et al. Role of COL4A1 in small-vessel disease and hemorrhagic stroke. N Engl J Med 2006;354:1489-1496.

30. Gould DB, Phalan FC, Breedveld GJ, et al. Mutations in Col4a1 cause perinatal cerebral hemorrhage and porencephaly. Science 2005;308:1167-1171.

31. Volonghi I, Pezzini A, Del Zotto E, et al. Role of COL4A1 in basement-membrane integrity and cerebral small-vessel disease. The COL4A1 stroke syndrome. Curr Med Chem 2010;17:13171324

32. Lanfranconi S, Markus HS. COL4A1 mutations as a monogenic cause of cerebral small vessel disease: a systematic review. Stroke 2010;41:e513-518.

33. Caplan LR, Arenillas J, Cramer SC, et al. Stroke-Related Translational Research. Archives of Neurology 2011. doi:10.1001/arch neurol.2011.99

34. Lanktree MB, Dichgans M, Hegele RA. Advances in genomic analysis of stroke: what have we learned and where are we headed? Stroke 2010;41:825-832.

35. Brown MB, Biffi A, Jeremiasz J, et al. International Stroke Conference and Nursing Symposium Poster Presentations:
Genome Wide Association Study of Intracerebral Hemorrhage. Stroke 2011;42:e218.

36. Woo D. Ethnic/racial variations of intracerebral hemorrhage (ERICH). [Cited 2011 February 28]; available from: http:// clinicaltrials.gov/ct2/show/NCT01202864; NLM Identifier: NCT01202864. Accessed February 28.

37. Furlan AJ, Whisnant JP, Elveback LR. The decreasing incidence of primary intracerebral hemorrhage: a population study. Ann Neurol 1979;5:367-373

38. Ariesen MJ, Claus SP, Rinkel GJ, Algra A. Risk factors for intracerebral hemorrhage in the general population: a systematic review. Stroke 2003;34:2060-2065.

39. SHEP Cooperative Research Group. Prevention of stroke by antihypertensive drug treatment in older persons with isolated systolic hypertension. Final results of the Systolic Hypertension in the Elderly Program (SHEP). JAMA 1991;265:3255-3264.

40. Mutlu N, Berry RG, Alpers BJ. Massive Cerebral Hemorrhage. Clinical and Pathological Correlations. Arch Neurol 1963;8:644 661.

41. Broderick J, Brott T, Tomsick T, Leach A. Lobar hemorrhage in the elderly. The undiminishing importance of hypertension. Stroke 1993;24:49-51.

42. Charcot JM, Bouchard C. Nouvelles recherché sur la pathogenie de l'hemorragie cerebrale. Arch Physiol Norm Pathol 1868. 1: p. 110.

43. Johansson BB. Vascular mechanisms in hypertensive cerebrovascular disease. J Cardiovasc Pharmacol 1992;(19 suppl 3):S11-S15.

44. Ellis AG. The pathogenesis of spontaneous cerebral hemorrhage. Proc Pathol Soc (Phila) 1909;12:197.

45. Qureshi AI, Tuhrim S, Broderick JP, Batjer HH, Hondo H, Hanley DF. Spontaneous intracerebral hemorrhage. N Engl J Med 2001;344:1450-1460.

46. Fisher CM. Pathological observations in hypertensive cerebral hemorrhage. J Neuropathol Exp Neurol 1971;30:536-550.

47. Broderick JP, Brott TG, Tomsick T, Barsan W, Spilker J. Ultraearly evaluation of intracerebral hemorrhage. J Neurosurg 1990;72:195-199.

48. Kazui S, Naritomi H, Yamamoto H, Sawada T, Yamaguchi T. Enlargement of spontaneous intracerebral hemorrhage. Incidence and time course. Stroke 1996;27:1783-1787.

49. Brott T, Broderick J, Kothari R, et al. Early hemorrhage growth in patients with intracerebral hemorrhage. Stroke 1997;28:1-5.

50. Fujii Y, Takeuchi S, Sasaki O, Minakawa T, Tanaka R. Multivariate analysis of predictors of hematoma enlargement in spontaneous intracerebral hemorrhage. Stroke 1998;29:1160 1166.

51. Jauch EC, Lindsell CJ, Adeoye O, et al. Lack of evidence for an association between hemodynamic variables and hematoma growth in spontaneous intracerebral hemorrhage. Stroke 2006;37:2061-2065.

52. Fehr MA, Anderson DC. Incidence of progression or rebleeding in hypertensive intracerebral hemorrhage. J Stroke Cerebrovascular Dis 1991;1:111-116.

53. Silva Y, Leira R, Tejada J, Lainez JM, Castillo J, Davalos A. Molecular signatures of vascular injury are associated with early growth of intracerebral hemorrhage. Stroke 2005;36:86-91.

54. Davis SM, Broderick J, Hennerici M, et al. Hematoma growth is a determinant of mortality and poor outcome after intracerebral hemorrhage. Neurology 2006;66:1175-1181.

55. Mayer SA. Recombinant activated factor VII for acute intracerebral hemorrhage. Stroke 2007;38(2 suppl):763-767.

56. Mayer SA, Brun NC, Begtrup K, et al. Efficacy and safety of recombinant activated factor VII for acute intracerebral hemorrhage. N Engl J Med 2008;358:2127-2137.

57. Delgado Almandoz JE, Yoo AJ, Stone MJ, et al. Systematic characterization of the computed tomography angiography spot sign in primary intracerebral hemorrhage identifies patients at 
highest risk for hematoma expansion: the spot sign score. Stroke 2009;40:2994-3000.

58. Goldstein JN, Fazen LE, Snider R, et al. Contrast extravasation on CT angiography predicts hematoma expansion in intracerebral hemorrhage. Neurology 2007;68:889-894.

59. Wada R, Aviv RI, Fox AJ, et al. CT angiography "spot sign" predicts hematoma expansion in acute intracerebral hemorrhage. Stroke 2007;38:1257-1262.

60. Hallevi H, Abraham AT, Barreto AD, Grotta JC, Savitz SI. The spot sign in intracerebral hemorrhage: the importance of looking for contrast extravasation. Cerebrovasc Dis 2010;29:217-220.

61. Flaherty M, Jauch E. The Spot Sign for Predicting and Treating ICH Growth Study (STOP-IT) 2008; Available from: http://clinicaltrials. gov/ct2/show/NCT00810888?term=STOP-IT\&rank=1; NLM Identifier: NCT00810888. Accessed Jan 1.

62. Willmot M, Leonardi-Bee J, Bath PM. High blood pressure in acute stroke and subsequent outcome: a systematic review. Hypertension 2004;43:18-24.

63. Zhang Y, Reilly KH, Tong W, et al. Blood pressure and clinical outcome among patients with acute stroke in Inner Mongolia, China. J Hypertens 2008;26:1446-1452.

64. Ohwaki K, Yano E, Nagashima H, Hirata M, Nakagomi T, Tamura A. Blood pressure management in acute intracerebral hemorrhage: relationship between elevated blood pressure and hematoma enlargement. Stroke 2004;35:1364-1367.

65. Zazulia AR, Diringer MN, Videen TO, et al. Hypoperfusion without ischemia surrounding acute intracerebral hemorrhage. J Cereb Blood Flow Metab 2001;21:804-810.

66. Anderson CS, Huang Y, Wang JG, et al. Intensive blood pressure reduction in acute cerebral haemorrhage trial (INTERACT): a randomised pilot trial. Lancet Neurol 2008;7:391-399.

67. Anderson C. The Second Intensive Blood Pressure Reduction in Acute Cerebral Haemorrhage Trial (INTERACT2). Available from: http://clinicaltrials.gov/ct2/show/NCT00716079; NLM Identifier: NCT00716079.

68. Qureshi AI. Antihypertensive treatment of acute cerebral hemorrhage. Crit Care Med 2010;38:637-648.

69. Qureshi AI. Antihypertensive Treatment in Acute Cerebral Hemorrhage-II (ATACH-II). 2010 [cited 2011 May 20]; Available from: http://clinicaltrials.gov/ct2/show/NCT01176565; NLM Identifier: NCT01176565. Accessed May 20.

70. Mayer SA, Sacco RL, Shi T, Mohr JP. Neurologic deterioration in noncomatose patients with supratentorial intracerebral hemorrhage. Neurology 1994;44:1379-1384.

71. Qureshi AI, Suri MF, Ostrow PT, et al. Apoptosis as a form of cell death in intracerebral hemorrhage. Neurosurgery 2003;52: 1041-1048.

72. Wu G, Xi G, Huang F. Spontaneous intracerebral hemorrhage in humans: hematoma enlargement, clot lysis, and brain edema. Acta Neurochir Suppl 2006;96:78-80.

73. Ruscalleda J, Peiro A. Prognostic factors in intraparenchymatous hematoma with ventricular hemorrhage. Neuroradiology 1986; 28:34-37.

74. Welch KMA, Caplan LR, Reis DJ, Seisjo BK, B W, eds. Primer on Cerebrovascular Diseases. 1997, Academic Press.

75. Xi G, Hua Y, Bhasin RR, Ennis SR, Keep RF, Hoff JT. Mechanisms of edema formation after intracerebral hemorrhage: effects of extravasated red blood cells on blood flow and bloodbrain barrier integrity. Stroke 2001;32:2932-2938.

76. Lee KR, Kawai N, Kim S, Sagher O, Hoff JT. Mechanisms of edema formation after intracerebral hemorrhage: effects of thrombin on cerebral blood flow, blood-brain barrier permeability, and cell survival in a rat model. J Neurosurg 1997;86:272-278.

77. Rosenberg GA, Navratil M. Metalloproteinase inhibition blocks edema in intracerebral hemorrhage in the rat. Neurology 1997;48:921-926.
78. Abilleira S, Montaner J, Molina CA, Monasterio J, Castillo J, Alvarez-Sabin J. Matrix metalloproteinase-9 concentration after spontaneous intracerebral hemorrhage. J Neurosurg 2003;99:6570.

79. Makogonenko E, Tsurupa G, Ingham K, Medved L. Interaction of fibrin(ogen) with fibronectin: further characterization and localization of the fibronectin-binding site. Biochemistry 2002;41:7907-7913.

80. Hickenbottom SL, Grotta JC, Strong R, Denner LA, Aronowski J. Nuclear factor-kappaB and cell death after experimental intracerebral hemorrhage in rats. Stroke 1999;30:2472-2478.

81. Gong C, Boulis N, Qian J, Turner DE, Hoff JT, Keep RF. Intracerebral hemorrhage-induced neuronal death. Neurosurgery 2001;48:875-883.

82. Mendelow AD, Gregson BA, Fernandes HM, et al. Early surgery versus initial conservative treatment in patients with spontaneous supratentorial intracerebral haematomas in the International Surgical Trial in Intracerebral Haemorrhage (STICH): a randomised trial. Lancet 2005;365:387-397.

83. Hankey GJ, Hon C. Surgery for primary intracerebral hemorrhage: is it safe and effective? A systematic review of case series and randomized trials. Stroke 1997;28:2126-2132.

84. Mendelow AD. Surgical trial in lobar intracerebral hemorrhage. 2006; available from: http://www.strokecenter.org/trials/trialDetail.aspx?tid $=724 \&$ search_string $=$ stich.

85. Vespa P, McArthur D, Miller C, et al. Frameless stereotactic aspiration and thrombolysis of deep intracerebral hemorrhage is associated with reduction of hemorrhage volume and neurological improvement. Neurocrit Care 2005;2:274-281.

86. Wagner KR, Beiler S, Beiler C, et al. Delayed profound local brain hypothermia markedly reduces interleukin-1beta gene expression and vasogenic edema development in a porcine model of intracerebral hemorrhage. Acta Neurochir Suppl 2006;96:177-182.

87. Mohr JP, Choi DW, Grotta JC, Weir B, PA W, eds. Stroke: Pathophysiology, Diagnosis, and Management. 1998, Churchill Livingstone.

88. Findlay JM, Weir BK, Kanamaru K, et al. Intrathecal fibrinolytic therapy after subarachnoid hemorrhage: dosage study in a primate model and review of the literature. Can J Neurol Sci 1989;16:28-40.

89. Morgan T, Awad I, Keyl P, Lane K, Hanley D. Preliminary report of the clot lysis evaluating accelerated resolution of intraventricular hemorrhage (CLEAR-IVH) clinical trial. Acta Neurochir Suppl 2008;105:217-220.

90. Aronowski J, Hall CE. New horizons for primary intracerebral hemorrhage treatment: experience from preclinical studies. Neurol Res 2005;27:268-279.

91. Xue M, Del Bigio MR. Intracerebral injection of autologous whole blood in rats: time course of inflammation and cell death. Neurosci Lett 2000;283:230-232.

92. Cox G, Crossley J, Xing Z. Macrophage engulfment of apoptotic neutrophils contributes to the resolution of acute pulmonary inflammation in vivo. Am J Respir Cell Mol Biol 1995;12:232237.

93. Haslett C. Granulocyte apoptosis and its role in the resolution and control of lung inflammation. Am J Respir Crit Care Med 1999;160(5 pt 2):S5-S11.

94. Batchelor PE, Liberatore GT, Wong JY, et al. Activated macrophages and microglia induce dopaminergic sprouting in the injured striatum and express brain-derived neurotrophic factor and glial cell line-derived neurotrophic factor. J Neurosci 1999;19:1708-1716.

95. Lin TN, Cheung WM, Wu JS, et al. 15d-prostaglandin J2 protects brain from ischemia-reperfusion injury. Arterioscler Thromb Vasc Biol 2006;26:481-487. 
96. Majno G. The Healing Hand: Man and Wound in the Ancient Word, 1975. Cambridge, MA: Harvard University Press.

97. Wang J, Rogove AD, Tsirka AE, Tsirka SE. Protective role of tuftsin fragment $1-3$ in an animal model of intracerebral hemorrhage. Ann Neurol 2003;54:655-664.

98. Yenari MA, Xu L, Tang XN, Qiao Y, Giffard RG. Microglia potentiate damage to blood-brain barrier constituents: improvement by minocycline in vivo and in vitro. Stroke 2006;37:1087-1093.

99. Yang GY, Betz AL, Chenevert TL, Brunberg JA, Hoff JT. Experimental intracerebral hemorrhage: relationship between brain edema, blood flow, and blood-brain barrier permeability in rats. J Neurosurg 1994;81:93-102.

100. Hattori R, Inoue R, Sase K, et al. Preferential inhibition of inducible nitric oxide synthase by ebselen. Eur $\mathrm{J}$ Pharmacol 1994;267:R1-R2.

101. Xi G, Keep RF, Hoff JT. Erythrocytes and delayed brain edema formation following intracerebral hemorrhage in rats. J Neurosurg 1998;89:991-996.

102. Zhao X, Sun G, Zhang J, et al. Hematoma resolution as a target for intracerebral hemorrhage treatment: role for peroxisome proliferator-activated receptor gamma in microglia/macrophages. Ann Neurol 2007;61:352-362.

103. Zhao X, Grotta J, Gonzales N, Aronowski J. Hematoma resolution as a therapeutic target: the role of microglia/macrophages. Stroke 2009;40(3 suppl):S92-S94.

104. Gonzales NR. Safety of Pioglitazone for Hematoma Resolution In Intracerebral Hemorrhage (SHRINC). 2009 [Cited 2011 May 20]; available from: http://clinicaltrials.gov/ct2/show/NCT00827892; NLM Identifier: NCT00827892. Accessed May 20.

105. Chu K, Jeong SW, Jung KH, et al. Celecoxib induces functional recovery after intracerebral hemorrhage with reduction of brain edema and perihematomal cell death. J Cereb Blood Flow Metab 2004;24:926-933.

106. Park HK, Lee SH, Chu K, Roh JK. Effects of celecoxib on volumes of hematoma and edema in patients with primary intracerebral hemorrhage. J Neurol Sci 2009;279(1-2):43-46.

107. Belayev L, Saul I, Busto R, et al. Albumin treatment reduces neurological deficit and protects blood-brain barrier integrity after acute intracortical hematoma in the rat. Stroke 2005;36:326-331.

108. Zoellner H, Hofler M, Beckmann R, et al. Serum albumin is a specific inhibitor of apoptosis in human endothelial cells. J Cell Sci 1996;109(pt 10):2571-2580.

109. Belayev L, Obenaus A, Zhao W, et al. Experimental intracerebral hematoma in the rat: characterization by sequential magnetic resonance imaging, behavior, and histopathology. Effect of albumin therapy. Brain Res 2007;1157:146-155.

110. University G. Albumin for Intracerebral Hemorrhage Intervention (ACHIEVE). [Cited December 21, 2010]; available from: http://clinicaltrials.gov/ct2/show/NCT00990509; NLM Identifier: NCT00990509. Accessed December 21.

111. Zhao Z, Cheng M, Maples KR. et al. NXY-059, a novel free radical trapping compound, reduces cortical infarction after permanent focal cerebral ischemia in the rat. Brain Res 2001;909:46.

112. Sydserff SG, Borelli AR, Green AR, Cross AJ. Effect of NXY059 on infarct volume after transient or permanent middle cerebral artery occlusion in the rat; studies on dose, plasma concentration and therapeutic time window. $\mathrm{Br} \mathrm{J}$ Pharmacol 2002;135:103-112.

113. Lyden PD, Shuaib A, Lees KR, et al. Safety and tolerability of NXY-059 for acute intracerebral hemorrhage: the CHANT Trial. Stroke 2007;38:2262-2269.

114. Saver JL, Albers GW, Dunn B, Johnston KC, Fisher M. Stroke Therapy Academic Industry Roundtable (STAIR) recommendations for extended window acute stroke therapy trials. Stroke 2009;40:2594-2600.
115. Wu H, Wu T, Xu X, Wang J, Wang J. Iron toxicity in mice with collagenase-induced intracerebral hemorrhage. J Cereb Blood Flow Metab 2010;31(5):1243-1250.

116. Gu Y, Hua Y, Keep RF, Morgenstern LB, Xi G. Deferoxamine reduces intracerebral hematoma-induced iron accumulation and neuronal death in piglets. Stroke 2009;40:2241-2243.

117. Okauchi M, Hua Y, Keep RF, Morgenstern LB, Xi G. Effects of deferoxamine on intracerebral hemorrhage-induced brain injury in aged rats. Stroke 2009;40:1858-1863.

118. Okauchi M, Hua Y, Keep RF, Morgenstern LB, Schallert T, Xi G. Deferoxamine treatment for intracerebral hemorrhage in aged rats: therapeutic time window and optimal duration. Stroke 2010;41:375-382.

119. Nakamura T, Keep RF, Hua Y, Schallert T, Hoff JT, Xi G. Deferoxamine-induced attenuation of brain edema and neurological deficits in a rat model of intracerebral hemorrhage. Neurosurg Focus 2003;15:ECP4.

120. Selim M. Dose Finding and Safety Study of Deferoxamine in Patients With Brain Hemorrhage (DFO In ICH). [Cited December 21, 2010]; available from: http://clinicaltrials.gov/ct2/show/NCT00598572; NLM Identifier: NCT00598572. Accessed December 21.

121. Selim M, Goldstein JN, Gomes J, et al. Safety and Tolerability of Deferoxamine in Acute Cerebral Hemorrhage: DFO in ICH Study. In International Stroke Conference, 2011. Los Angeles, CA.

122. Sturgeon JD, Folsom AR, Longstreth WT, Jr., Shahar E, Rosamond WD, Cushman M. Risk factors for intracerebral hemorrhage in a pooled prospective study. Stroke 2007;38:2718-2725.

123. Orken DN, Kenangil G, Celik M, et al. Association of low cholesterol with primary intracerebral haemorrhage: a case control study. Acta Neurol Scand 2009;119:151-154.

124. Amarenco P, Bogousslavsky J, Callahan A, 3rd, et al. High-dose atorvastatin after stroke or transient ischemic attack. N Engl $\mathrm{J}$ Med 2006;355:549-559.

125. Sironi L, Cimino M, Guerrini U, et al. Treatment with statins after induction of focal ischemia in rats reduces the extent of brain damage. Arterioscler Thromb Vasc Biol 2003;23:322-327.

126. Laufs U, Gertz K, Dirnagl U, Bohm M, Nickenig G, Endres M. Rosuvastatin, a new HMG-CoA reductase inhibitor, upregulates endothelial nitric oxide synthase and protects from ischemic stroke in mice. Brain Res 2002;942(1-2):23-30.

127. Amin-Hanjani S, Stagliano NE, Yamada M, Huang PL, Liao JK, Moskowitz MA. Mevastatin, an HMG-CoA reductase inhibitor, reduces stroke damage and upregulates endothelial nitric oxide synthase in mice. Stroke 2001;32:980-986.

128. Seyfried D, Han Y, Lu D, Chen J, Bydon A, Chopp M. Improvement in neurological outcome after administration of atorvastatin following experimental intracerebral hemorrhage in rats. J Neurosurg 2004;101:104-107.

129. Jung $\mathrm{KH}$, Chu $\mathrm{K}$, Jeong $\mathrm{SW}$, et al. HMG-CoA reductase inhibitor, atorvastatin, promotes sensorimotor recovery, suppressing acute inflammatory reaction after experimental intracerebral hemorrhage. Stroke 2004;35:1744-1749.

130. Karki K, Knight RA, Han Y, et al. Simvastatin and atorvastatin improve neurological outcome after experimental intracerebral hemorrhage. Stroke 2009;40:3384-3389.

131. Zacco A, Togo J, Spence K, et al. 3-hydroxy-3-methylglutaryl coenzyme A reductase inhibitors protect cortical neurons from excitotoxicity. J Neurosci 2003;23:11104-11111.

132. Naval NS, Abdelhak TA, Urrunaga N, Zeballos P, Mirski MA, Carhuapoma JR. An association of prior statin use with decreased perihematomal edema. Neurocrit Care 2008;8:13-18.

133. Tapia-Perez H, Sanchez-Aguilar M, Torres-Corzo JG, et al. Use of statins for the treatment of spontaneous intracerebral hemorrhage: results of a pilot study. Cen Eur Neurosurg 2009;70: $15-20$. 
134. University JH. Simvastatin For Intracerebral Hemorrhage Study. [Cited 2010 December 21]; available from: http:// clinicaltrials.gov/ct2/show/NCT00718328; NLM Identifier: NCT00718328. Accessed December 21.

135. Saloheimo P, Ahonen M, Juvela S, Pyhtinen J, Savolainen ER, Hillbom M. Regular aspirin-use preceding the onset of primary intracerebral hemorrhage is an independent predictor for death. Stroke 2006;37:129-133.

136. Toyoda K, Okada Y, Minematsu K, et al. Antiplatelet therapy contributes to acute deterioration of intracerebral hemorrhage. Neurology 2005;65:1000-1004.

137. Naidech AM, Jovanovic B, Liebling S, et al. Reduced platelet activity is associated with early clot growth and worse 3-month outcome after intracerebral hemorrhage. Stroke 2009;40:23982401.

138. Foerch C, Sitzer M, Steinmetz H, Neumann-Haefelin T. Pretreatment with antiplatelet agents is not independently associated with unfavorable outcome in intracerebral hemorrhage. Stroke 2006;37:2165-2167.

139. Sansing LH, Messe SR, Cucchiara BL, Cohen SN, Lyden PD, Kasner SE. Prior antiplatelet use does not affect hemorrhage growth or outcome after ICH. Neurology 2009;72:13971402 .

140. Thompson BB, Bejot Y, Caso V, et al. Prior antiplatelet therapy and outcome following intracerebral hemorrhage: a systematic review. Neurology 2010;75:1333-1342.

141. Creutzfeldt CJ, Weinstein JR, Longstreth WT, Jr., Becker KJ, McPharlin TO, Tirschwell DL. Prior antiplatelet therapy, platelet infusion therapy, and outcome after intracerebral hemorrhage. J Stroke Cerebrovasc Dis 2009;18:221-228.

142. Illoh K, Lucke J, Illoh O. Intracerebral Hemorrhage Patients Who Receive Blood Products have Worse Outcomes. Stroke: Abstracts From the 2008 International Stroke Conference 2008;39:709.

143. Oulu Uo, University H. Platelet Transfusion in Acute Intracerebral Hemorrhage. [cited October 21, 2010]; available from: http://clinicaltrials.gov/ct2/show/NCT00699621; NLM Identifier: NCT00699621. Accessed October 21.
144. University N. Improving Platelet Activity for Cerebral Hemorrhage Treatment - DDAVP Proof of Concept (IMPACT). [Cited 2010 December 21]; available from: http://clinicaltrials.gov/ct2/ show/NCT00961532; NLM Identifier: NCT00961532. Accessed December 21.

145. Poller L, Jespersen J, Ibrahim S. Dabigatran versus warfarin in patients with atrial fibrillation. N Engl J Med 2009;361:26732675.

146. Flaherty ML, Tao H, Haverbusch M, et al. Warfarin use leads to larger intracerebral hematomas. Neurology 2008;71:1084-1089.

147. Morgenstern LB, Hemphill JC, 3rd, Anderson C, et al. Guidelines for the management of spontaneous intracerebral hemorrhage: a guideline for healthcare professionals from the American Heart Association/American Stroke Association. Stroke 2010;41:21082129.

148. Kawai N, Kawanishi M, Okauchi M, Nagao S. Effects of hypothermia on thrombin-induced brain edema formation. Brain Res 2001;895:50-58.

149. MacLellan CL, Davies LM, Fingas MS, Colbourne F. The influence of hypothermia on outcome after intracerebral hemorrhage in rats. Stroke 2006;37:1266-1270.

150. Wu J, Yang S, Hua Y, Liu W, Keep RF, Xi G. Minocycline attenuates brain edema, brain atrophy and neurological deficits after intracerebral hemorrhage. Acta Neurochir Suppl 2010;106:147-150.

151. Auriat AM, Colbourne F. Delayed rehabilitation lessens brain injury and improves recovery after intracerebral hemorrhage in rats. Brain Res 2009;1251:262-268.

152. Auriat AM, Wowk S, Colbourne F. Rehabilitation after intracerebral hemorrhage in rats improves recovery with enhanced dendritic complexity but no effect on cell proliferation. Behav Brain Res 2010;214:42-47.

153. Takamatsu Y, Ishida A, Hamakawa M, Tamakoshi K, Jung CG, Ishida $\mathrm{K}$. Treadmill running improves motor function and alters dendritic morphology in the striatum after collagenase-induced intracerebral hemorrhage in rats. Brain Res 2010;1355:165-173.

154. Igor Antončić M. Pilot Study of Hypothermia for Intracerebral Hemorrhage in Croatia. http://clinicaltrials.gov/ct2/show/ NCT01221142. Accessed October 21. 